\title{
BMJ Open Enablers and barriers to effective clinical supervision in the workplace: a rapid evidence review
}

\author{
Charlotte Rothwell (D) , ${ }^{1}$ Amelia Kehoe (D) , ${ }^{2}$ Sophia Farhene Farook, ${ }^{3}$ Jan Illing (i) ${ }^{4}$
}

To cite: Rothwell C, Kehoe A, Farook SF, et al. Enablers and barriers to effective clinical supervision in the workplace: a rapid evidence review. BMJ Open 2021;11:e052929. doi:10.1136/ bmjopen-2021-052929

- Prepublication history and additional supplemental material for this paper are available online. To view these files, please visit the journal online (http://dx.doi.org/10.1136/ bmjopen-2021-052929).

Received 06 May 2021 Accepted 26 August 2021

Check for updates

(c) Author(s) (or their employer(s)) 2021. Re-use permitted under CC BY-NC. No commercial re-use. See rights and permissions. Published by BMJ.

${ }^{1}$ School of Medical Education, Newcastle University, Newcastle upon Tyne, UK

${ }^{2}$ Health Professions Education Unit, Hull York Medical School, York, UK

${ }^{3}$ Emergency Medicine, Newcastle upon Tyne Hospitals NHS Trust, Newcastle upon Tyne, UK

${ }^{4}$ Health Professions Education Centre, Royal College of Surgeons in Ireland, Dublin, Ireland

Correspondence to Dr Charlotte Rothwell; charlotte.rothwell@ncl.ac.uk

\begin{abstract}
Objectives We aimed to review the international literature to understand the enablers of and barriers to effective clinical supervision in the workplace and identify the benefits of effective clinical supervision.

Design A rapid evidence review.

Data sources Five databases (CINAHL, OVID Embase, OVID Medline, OVID PsycInfo and ProQuest) were searched to ensure inclusion and breadth of healthcare professionals.

Eligibility criteria Studies identifying enablers and barriers to effective clinical supervision across healthcare professionals in a Western context between 1 January 2009 and 12 March 2019.
\end{abstract}

Data extraction and synthesis An extraction framework with a detailed inclusion/exclusion criteria to ensure rigour was used to extract data. Data were analysed using a thematic qualitative synthesis. These themes were used to answer the research objectives.

Results The search identified 15922 papers, reduced to 809 papers following the removal of duplicates and papers outside the inclusion criteria, with 135 papers being included in the full review. Enablers identified included regular supervision, occurs within protected time, in a private space and delivered flexibly. Additional enablers included supervisees being offered a choice of supervisor; supervision based on mutual trust and a positive relationship; a cultural understanding between supervisor and supervisee; a shared understanding of the purpose of supervision, based on individual needs, focused on enhancing knowledge and skills; training and feedback being provided for supervisors; and use of a mixed supervisor model, delivered by several supervisors, or by those trained to manage the overlapping (and potentially conflicting) needs of the individual and the service. Barriers included a lack of time, space and trust. A lack of shared understanding to the purpose of the supervision, and a lack of ongoing support and engagement from leadership and organisations were also found to be barriers to effective clinical supervision.

Conclusions This review identified several enablers of and barriers to effective clinical supervision and the subsequent benefits of effective clinical supervision in a healthcare setting.

\section{INTRODUCTION}

Understanding what makes clinical supervision effective and learning more about the

\section{Strengths and limitations of this study}

This paper was based on evidence identified in the international literature using a rapid review, which involves a systematic search and rigorous analysis.

- Papers were limited to Western only and the last 10 years for pragmatic reasons.

- Although in many areas there was a vast amount of information, which provides strength to the findings, a rapid review necessarily pays less attention to study design and sample sizes.

- Much of the data were heterogeneous in nature, and this also hindered our ability to relate the findings to specific professions and settings.

- The findings drawn from the overall themes were evident across much of the literature.

barriers to and challenges of effective supervision are important concerns for the health and social care workforce. Most organisations provide some provision, but many lack an understanding about why it is important, who should be involved, what the possible benefits are and how it could be improved.

Supervision is at the core of practice for all health and social care professionals, where there should be a sense of shared responsibility for the effectiveness and safety of practice. ${ }^{1}$ It is important to understand this complex process to ensure best practice for all participants involved (practitioner, service delivery manager, clinical supervisor, peers, clients and other service users, the profession itself).

Supervision has been described as an event that involves an ongoing professional relationship, between two and more staff members with different levels of knowledge or expertise, to support professional development and to enhance knowledge and skills. $^{2}$ Definitions of supervision emphasise the promotion of professional development in addition to ensuring patient safety. For example, Nancarrow et $a l^{2}$ focus on the progression of clinical practice through professional guidance and support and refer 
to Proctor's ${ }^{3}$ three functions of supervision-managerial/administrative, educational and supportive. All three functions should be overlapping and flexible. ${ }^{2}$

It has been suggested that there are many forms of supervision: internal managerial, internal reflective, external professional and external personal. ${ }^{14}$ At one end of this continuum, managerial supervision takes place inside the organisation and is mostly focused on task and process. At the other end, personal supervision is worker focused and centres mainly on the narrative brought into the supervision space by the worker. ${ }^{1}$ This last type of supervision (personal) has been highly valued by workers to air their feelings; providing a safe place to connect and self-reflect. Personal supervision allowed a more intensive focus on clinical issues and personal professional development rather than organisational concerns. ${ }^{4}$ Two types of supervision tend to coexist when the line manager is also the clinical supervisor-a focus on practitioner learning and development, and another focused on service delivery, risk management and underperformance. Kilminster and Jolly ${ }^{5}$ argued for clarity on dealing with underperformance in addition to identifying what helps and hinders effective clinical supervision. Managing this split highlights the need for supervisor training.

In this review, we used the following definition of supervision as it encompassed both personal development and service development in the context of a relationship extending over time:

This relationship is evaluative, extends over time and has the simultaneous purposes of enhancing the professional functioning of the more junior person and monitoring the quality of the professional services. (Bernard and Goodyear, p8) ${ }^{6}$

While it is evident that supervision is important, we must now understand exactly what aspects of supervision we should be focusing on, and it is hoped that best practice can be sought from looking across such a range of different healthcare professionals. Any critical differences that impacted on supervision across health professions were also noted.

\section{Identifying the focus of supervision}

The aim of this rapid review was: 1) to syntheses the evidence of international literature on the enablers of, and barriers to effective clinical supervision in the workplace; 2) to identify the benefits of effective clinical supervision in the workplace.

\section{METHODS}

A Rapid Evidence Assessment (REA) was used in this study. A REA is similar to a systematic review in that they both use rigorous methods of appraising and synthesising evidence from published studies. ${ }^{7}$ However, restrictions on the data retrieved are placed on the search at the data collection phase.

\section{Search strategy}

The research protocol was developed with advice from a data analyst at Newcastle University. As a result, we refined our initial search strategy and targeted the most appropriate databases. The following databases were used to ensure a breadth of health and social care professions were included: CINAHL, (Allied and Health Professionals literature), OVID Embase, OVID Medline (Medical literature), OVID PsycInfo, (Psychological literature) and ProQuest (Social Science literature). See the Search strategy section for a breakdown of search terms used.

A systematic search (see online supplemental material 1 for search strategy) of each database was carried out in line with our search strategy. As is typical of rapid reviews, limits were placed on the search to ensure the research could be done in a timely manner. For example, only including papers from the last 10 years ensured we were able to capture the most relevant documents for current supervision practice in a shorter space of time. Search terms were developed to include a comprehensive list of healthcare professionals, supervision types and forms of effectiveness. Restrictions were placed on the databases in line with our search strategy.

\section{Procedure for screening of data, data extraction and ensuring quality assurance}

All citations were downloaded to EndNote (reference management database) and duplication was removed $(n=2683)$. Authors independently reviewed the same 500 titles and abstracts to make sure that the same papers were being included/excluded. Any discrepancies were discussed and the inclusion/exclusion criteria were refined as needed (see box 1). All 13239 titles and abstracts were screened by two researchers (CR and AK).

\section{Box 1 Revised inclusion/exclusion criteria}

\section{Inclusion criteria for papers}

1. Papers that include clinical supervision and/or peer support in the workplace.

2. Papers that include a regulated healthcare profession.

3. Papers published within the last 10 years (1 January 2009-12 March 2019).

4. Papers that include primary research and systematic reviews.

5. Papers which are quantitative, qualitative or mixed methods.

6. Papers written in English.

7. Papers reporting on a Western culture setting.

\section{Exclusion criteria for papers}

1. Focus not on formal and structured clinical/peer supervision (by this we mean that the supervision was not a 'one off' event but must have some ongoing relationship, as detailed in the very definition of supervision).

2. Not in healthcare context.

3. University setting.

4. Not evidence based (eg, opinion pieces, letters or weak evidence).

5. Paper not written in English/outside review period.

6. Supervision of children/animals/patients.

7. Non-Western culture setting. 
A pilot data extraction exercise was conducted to ensure quality assurance. This exercise involved all four reviewers independently reading full papers and was repeated with a further 10 papers to check consistency of inclusion/ exclusion and data extraction. The data extraction framework was revised following this initial review of papers. The clear inclusion/exclusion criteria and detailed data extraction form were used to ensure rigour. The data extraction form has been added as online supplemental material 2. Regular meetings were held between all four reviewers to ensure quality was maintained and to discuss uncertainties or queries that arose from the papers, and it was during this phase that the definition of clinical supervision was identified.

\section{Synthesis of papers}

Once the data were entered onto the data extraction database (see online supplemental material 2 for the data extraction form), the data were analysed using a qualitative thematic synthesis, ${ }^{8}$ which is a useful approach when aiming to pull out common elements across the heterogeneous literature. These themes were used to answer the research aims.

\section{Patient and public involvement}

Patients or the public were not involved in the design, or conduct, or reporting, or dissemination plans of our research.

\section{Findings}

A full review of papers was conducted on 809 publications from nearly 16000 initially identified (see figure 1 ). The final number of included papers was 135, with 674 being excluded.

\section{Paper demographics}

Setting

A range of countries were represented within the included papers, with the majority being from Australia (38), the
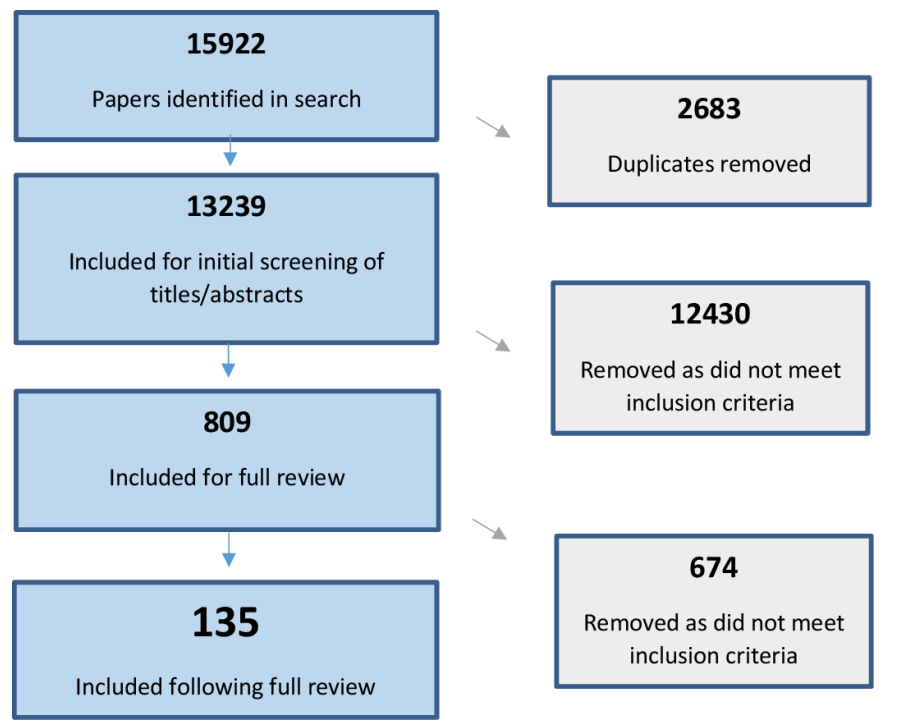

Figure 1 Flow chart of papers included in full review.
UK (31), the USA (24), New Zealand (11) and Canada (7). The findings were further diversified by the broad set of health and social care professions included in the review. The majority of papers included doctors, nurses, psychologists and social workers. Examples of other allied health professionals included were music therapists, physiotherapists, occupational therapists, speech and language therapists, podiatrists and dietitians.

\section{Research designs}

Papers included in the review were a mix of qualitative papers (53) using interview or focus group data and quantitative papers (50) using surveys and questionnaires. Several papers used a mixed-methods approach (15) and literature reviews (15), case study (1), action research (1), unidentified (1).

\section{Type of supervision}

Of the included papers, a large majority focused on clinical supervision (110), with a minority focusing on peer supervision (22) or both (3). These included both individual and group supervision sessions. Within the literature, there were several types of clinical supervision and peer supervision discussed. However, there was not always a clear distinction between different types of supervision, and terms were often used interchangeably such as peer supervision and peer mentoring. The working definition we used was clinical supervision, which was conducted either in a one-to-one or small group situation by a senior staff member or by a more experienced staff member at the same level. Clinical supervision included: action planning; reflection on clinical situations; role development and training; indirect and direct supervision; and included supervision from both internal and external organisations.

The research questions were answered using evidence from this literature review.

\section{What are the enablers to effective clinical supervision?} An open, supportive and safe environment

There was considerable evidence to highlight that having an open and safe environment where supervisees feel comfortable and trust their supervisor is an integral part of supervision. ${ }^{4-34}$ Having the time to discuss personal issues based on the needs of the individual was identified as an important focus for supervision. ${ }^{30} 32$ 35-37 There was also evidence of the value of time spent reflecting on practice, including ethical issues ${ }^{141518283839}$ and of receiving feedback. ${ }^{15} 3032$ 39-42

\section{Establishing a supervisory relationship based on trust}

Being able to develop a positive relationship with a supervisor that was based on trust was seen as key by a wide range of professions. ${ }^{2} 41011283942-46$ Supervisors who were deemed experts in their own profession were also more likely to be viewed as credible and trustworthy, and supervisees felt they were better placed to support them. ${ }^{11} 123246-48$ Trust was also underpinned by having the opportunity to be able to explore each other's belief and 
value systems in a neutral space, away from organisational hierarchies and the workplace and where emotions could be managed in an open and reflective way ${ }^{10} 49$ and when the supervisee respected the supervisor personally and professionally and both parties could self-disclose experiences. ${ }^{3250}$

\section{Regular supervision with timely feedback}

Many studies reported on the importance of receiving regular and constructive feedback during supervision $^{2141532404451-54}$ and having the time to reflect on practice. ${ }^{14151828425155}$ Supervision was valued for the sharing of tacit knowledge, for providing real-time feedback ${ }^{41}$ and when it provided confirmation that staff had done the right thing. ${ }^{15}$

The majority of the literature reviewed did not specify supervision frequency. There was scant evidence on how often clinical supervision should take place. ${ }^{56}$ However, Dilworth et $a \bar{l}^{7}$ reported that supervision should take place on a monthly basis to ensure sufficient support. Furthermore, McMahon and Errity ${ }^{27}$ reported that supervision that was less than fortnightly was insufficient and healthcare workers who spent at least $60 \mathrm{~min}$ in supervision perceived their supervision to be more effective. ${ }^{58}$

Supervisory relationships develop over time and are complex,${ }^{59}$ therefore supervision should not be a one-off activity, instead, it needs to be sustained over time and from early on in a career. ${ }^{60}$ However, the importance of providing unplanned discussion time to support emerging needs and ensure staff well-being was also identified. ${ }^{61}$

\section{Training for supervisors}

Supervisors need to have training in cultural awareness to enable them to be culturally competent. This was seen as an asset leading to improvements in communication, reflection and problem-solving. ${ }^{4} 1062$ Supervisors also need to be trained on listening skills ${ }^{30} 3263$ and helping supervisees to problem solve. ${ }^{64}$ Findings showed that it was important that the supervisor was able to not only provide feedback, but also receive it themselves. ${ }^{2} 1530324142$

\section{What are the benefits of effective clinical supervision? Job satisfaction and staff retention}

Several studies reported that effective supervision was found to have a positive impact on: staff retention, ${ }^{61} 65-67$ job satisfaction, ${ }^{136869}$ staff well-being ${ }^{63}$ 70-72 and perceptions of being valued. ${ }^{73}$ Wilson $e t a l^{22}$ found that feedback from supervisors facilitated learning and encouraged staff development. Continual Professional Development (CPD) and training for supervisors themselves were also found to increase retention. ${ }^{74}$ Regular supervision was found to increase staff retention. ${ }^{61}$ McMahon and Errity ${ }^{27}$ reported that greater supervision frequency, with regular progress reviews, was significantly related to positive outcomes.

\section{Reduced stress and anxiety}

Several studies found that supervision reduced stress and anxiety. ${ }^{15} 22$ 44 47 70-72 75-78 Evidence suggested that the reduction in stress and anxiety came about as supervision provided a medium for sharing skills, knowledge and resources, in a supportive environment. ${ }^{1544} 47$ A reduction in stress for supervisors was also found, following the provision of training and CPD support for supervisors. ${ }^{74}$ Studies reported that supervision helped participants to manage their feelings, ${ }^{446}$ also improving understanding of the importance of well-being and learning to help reflect on practice. ${ }^{72}$

\section{Better working environment}

Research highlighted that effective supervision and a supportive working environment can improve the uptake of workplace policies as supervisees understand the importance and reason for the policies. ${ }^{79}$ Better teamwork, relationships and more support in the workplace can also help with professional development. ${ }^{61} \mathrm{~A}$ study by Davis and Burke ${ }^{16}$ reported that supervision with nurse managers improved communication among staff and facilitated reflection, sharing ideas and problem-solving.

\section{Increased quality of care delivery}

Several studies made links with the provision of effective supervision and an increase in quality of care. ${ }^{162371727680-82}$ A study carried out by da Silva Pinheiro and de Carvalho ${ }^{76}$ reported group supervision with nurses had helped them to manage their feelings, which they linked to an increase in quality of care for their patients. Claridge $e t a l^{81}$ looked at whether direct supervision with resident doctors increased patient outcomes. Results showed that with direct supervision, there was a greater uptake of compliance with managerial protocols, and as a result patient outcomes were improved. ${ }^{81}$

\section{What are the barriers to effective clinical supervision? \\ Lack of time and heavy workloads}

One of the main barriers identified for effective supervision was a lack of time and heavy workloads. ${ }^{217} 21253541485773$ 83-98 Many studies reported that supervisors were unable to find time for supervision due to busy work environments, which ultimately restricted supervisor flexibility and quality when they did find the time. ${ }^{538299}$ Other studies reported a lack of opportunity and time for reflection within supervision, which left staff feeling that they had to 'figure things out' for themselves without adequate support. ${ }^{32} 84$

Many noted that supervision was not a priority, for both supervisor and supervisee. ${ }^{2760717294100}$ As a result, supervision was sometimes perceived to be a bonus, ${ }^{11}$ feeling that they were expected to not 'dwell' on stressful workplace issues. There was often an expectation that supervisors had the time to develop relationships and would take the time to complete the necessary paperwork prior to and following supervision, which could be time-consuming. ${ }^{80}$

A lack of adequate resources could lead to an overstretched workforce not being able to support each other effectively, and a decline in clinical supervision due to pressures on staff time. ${ }^{18}$ Kenny and Allenby ${ }^{60}$ discussed a 
lack of monetary incentives for supervision, affecting how supervision was perceived and whether it was provided or attended. Supervisees only wanted to attend supervision when it was within work time and when there was protected time for it. ${ }^{60}$

\section{Lack of staffing, shift working}

The type of clinical environment could facilitate or hinder clinical supervision. ${ }^{56}$ Key factors were organisation location, shift work patterns and work-environmental factors (quantitative demands, tempo, cognitive demands, influence at work and social support). Jelinek $e t a p^{51}$ discussed that there was a reduction in supervision levels during unsociable shift patterns. Supervision was dependent on service demands and was often not seen as a priority if there was insufficient staff numbers in busy environments. Differences seemed to not only reflect culture (regardless of policy asserting its importance) but also ease of access to supervision. For example, there was a lack of supervision outside day shifts or in rural communities with fewer staff despite the potential for increased need due to professional isolation. 23360101

\section{Lack of management/organisational support}

Organisational culture and attitude toward supervisory practice were found to be important, needing managerial support and buy-in. ${ }^{60} 101$ If management do not recognise the importance of supervision, it is unlikely it will become embedded into the organisational culture, and a lack of commitment from organisations and managers can act as a barrier to providing the time and resources required for effective supervision. 2731377393101102 In busy agency settings, supervision can often be neglected or deferred, to accommodate the latest crisis, unless it is made a priority by management. ${ }^{1}$ A study exploring which nurses decided to participate in clinical supervision found that support from empowering and fair leadership was crucial, affecting the adoption and uptake of clinical supervision, both positively and negatively. ${ }^{103}$

\section{Lack of supervisor training and support}

Several studies reported that a lack of training for supervisors was a barrier and resulted in ineffective supervision. ${ }^{32} 3864769192$ 104-107 Supervision was varied and individual when no direction about how to approach it was available. ${ }^{56}$ Studies also reported a lack of quality in supervision when supervisors were unfamiliar with professional guidelines (ie, standards set by regulators), their role and responsibilities as a supervisor, ethical standards set in place by employers and inadequate educational preparation. ${ }^{11}$

Lack of supervisor competence and skills was identified in a number of studies highlighting barriers to effective clinical supervision, such as: being intolerant, blameful and inflexible, ${ }^{2}$ being unable to deal with unmotivated supervisees $^{7687}$ and manage differing personality types, ${ }^{108}$ the lack of ability to share feelings, ${ }^{49}$ inability to give appropriate feedback ${ }^{51}$ and an inability to understand personal issues. ${ }^{35}$

Lack of understanding and support when dealing with underperformance

Supervision should facilitate learning opportunities when needed. ${ }^{109}$ However, supervisors do not always have the time and opportunity to upskill staff or work with those who are underperforming. ${ }^{110}$ Kilbertus $e t ~ a l^{111}$ also found that some supervisors reported not feeling able or comfortable in recognising and managing a failing trainee. Issues arose when either the supervisor or supervisees were unaware of the supervisee's lack of knowledge and skills. ${ }^{43} 47$

A lack of support from employers was noted by supervisors when raising concerns about staff, ${ }^{51}$ not always being told where to signpost supervisees to if there were any concerns or needs outside of their remit (eg, mental health support). Supervisors themselves may also need to seek support. ${ }^{60}$ Supervisors also feared that if they gave supervisees negative feedback, that in turn they would receive negative teaching evaluations, and this would impact on their own future promotion and career. ${ }^{41}$ There was also evidence that clinical supervision was delegated to the most junior consultants, with the least experience to deal with complex underperforming trainees. ${ }^{110}$ Kilbertus $e t a l^{111}$ highlighted that a lack of continuity of feedback meant that it was easy for struggling residents to fall through the net.

\section{Dealing with supervision from another discipline or from an external organisation}

External supervisors (who work in a different organisation to their supervisee) and interprofessional supervision (supervisors from a different profession) may require additional training and guidance. ${ }^{1460}$ Interprofessional supervision can sometimes lead to misunderstanding due to differences in roles, responsibilities and levels of training. There may also be an absence of shared theory, language, differences in professional decisionmaking processes and codes of conduct. ${ }^{112}$ In addition, an oversight of ethical practice could be weaker with an interprofessional model. ${ }^{113}$ It may also disadvantage supervisors with regard to the professional role, not being able to raise all issues, and causes disempowerment due to differences in professional status ${ }^{1}$ and places a burden of responsibility on the supervisor to have a good working knowledge of the context of practice of other professions.

Beddoe $^{1}$ states that an external supervisor will hold less information about the practitioner compared with an internal supervisor, who will likely identify managerial concerns more effectively. Having an external supervisor, however, increased the likelihood that supervision took place. ${ }^{9114} 115$ Yet it was the supervisee who mainly set the agenda with regard to issues to be discussed, and therefore underperformance was more likely to remain concealed. This type of supervision highlights the 
weakness of self-assessment, which is a particular concern for those who are underperforming. ${ }^{116}$

\section{Lack of relationship and trust}

Supervisees need to feel that they can trust their supervisor, ${ }^{52}$ yet sadly, this was sometimes lacking. ${ }^{16} 176184117118$ Unhelpful and untrusting relationships led participants to distrust their supervisor's advice, or be self-critical. ${ }^{39} 52$ Palmer-Olsen $e t a l^{44}$ found that supervisors who did not establish a secure supervisory alliance were less effective in helping their supervisees learn to implement a specific therapy. A lack of supervisor commitment, or when supervision was reduced to a 'tick box' exercise, or too bureaucratic, it was found to be less effective. ${ }^{261}{ }^{85}$ It was also noted that sometimes people did not 'fit' with their supervisor. ${ }^{32} 39$

Lack of understanding about what supervision was and its purpose Several studies reported a lack of a common understanding about the role and purpose of supervision. ${ }^{26097100119} \mathrm{On}$ such occasions, supervisees reported anxiety and sometimes perceived that supervision equated to surveillance. ${ }^{11} 32446073120$ Negative associations with the term 'clinical supervision' also led to a lack of engagement. ${ }^{1137}$

\section{DISCUSSION}

This rapid systematic review aimed to identify the enablers of and barriers to effective clinical supervision and identified the benefits of supervision for supervisees and supervisors within the workplace.

When in place and done well, clinical supervision has many benefits for the organisation, professional development and patient services, and each of these three levels makes an important contribution to ensure benefit is achieved. This review has highlighted evidence which indicates what needs to be in place to ensure clinical supervision is effective. Evidence from the literature review indicates that the organisation plays a key role in ensuring supervision takes place,${ }^{6095102}$ that it is valued and expected, ${ }^{7395}$ that supervisors are trained ${ }^{293768799199} 104121$ and time is protected. ${ }^{22} 25799299122$ Supervision needs to be provided in a neutral, open, supportive environment to facilitate discussion and reflection on clinical practice, career development and any personal issues that may arise in the workplace. ${ }^{4} 910$ 13-16 182021 23-26 29 30 32-34 44117 123-125

Having a relationship based on trust with the supervisor was also found to be key. ${ }^{24101128323942-46}$ There was also evidence on the benefit of reflecting on practice ${ }^{1828}$ and on receiving feedback. ${ }^{30} 4041$ Having regular but flexible supervision that fitted around all stakeholders' needs was also highlighted as important. Clinical supervision provides the chance to facilitate learning opportunities when needed ${ }^{98}$ and to upskill staff who were underperforming. ${ }^{110}$

There was much evidence about the positive benefits of clinical supervision, in that those who received support through clinical supervision were better able to cope with the demands of the job ${ }^{23} 75$ and were less likely to leave. $^{69} 126$ Effective supervision increased resilience ${ }^{78}$ and job satisfaction. ${ }^{68} 69127$ There was also evidence to suggest that supervision helped with reducing stress and anxiety ${ }^{63}$ Supervision was also seen to drive up the quality of care and has a positive effect on the working environment. ${ }^{1623717780-8287}$

A number of barriers were highlighted within the literature that should be taken into consideration when exploring how to implement effective supervision practice. These included a lack of time and heavy workload,,$^{2} 16 \quad 1721 \quad 35 \quad 41 \quad 48 \quad 54 \quad 57 \quad 64 \quad 73 \quad 83-85 \quad 87-96 \quad 98$ a lack of resources, ${ }^{18} 3760$ unsupportive management and colleagues, ${ }^{2} 27317393102128$ a lack of supervisor training, ${ }^{11} 17323864769192$ 105-107 124 and a lack of trusting relationships and ongoing support. ${ }^{16} 1745617584117118$ Supervisees were also sometimes unaware of the purpose of the supervision practice, ${ }^{2} 6064100119$ impacting on engagement. ${ }^{2}$ A recent study has highlighted the need for supervision to include patient care, concluding that the usual model of meeting for a supervisory discussion away from patient care was not found to be effective. ${ }^{129}$ Although this is an interesting and important finding, our findings would suggest that the overall supervisory experience is not as simplistic as this. There is a need to take into account all of the factors and levels presented in this paper, there being no single answer leading to effective supervision.

There were no critical differences identified across the range of healthcare professionals in terms of ensuring effective supervision is in place, with similar themes being apparent across all. Naturally, professions such as psychologists and social workers will face different challenges and have different needs from their supervisory relationship; however, this is part of the supervisory process and identification of those needs is what will make it an effective experience for the supervisee.

It is clear from the evidence that support from management is needed to enable effective implementation, including cost and training for staff. However, this review has highlighted that supervision is subject to different interpretations by managers, who tend to focus more on service delivery rather than on staff development, and agreeing on the shared purpose of supervision is important to reduce ambiguity. ${ }^{110}$ Beddoe $^{1}$ argued that managerial supervision creates a shift from being practitioner focused to a monitoring agenda. Problems seem to arise when the focus was perceived to be monitoring performance, rather than on the provision of support. ${ }^{19103942115}$ Pack $^{72}$ highlights that line managers need to focus on protecting the employing organisation and their patients/clients from risk, ${ }^{96}$ whereas external supervisors can focus more on the personal development. This split may offer a solution that avoids the inevitable tension experienced by a manager who is also the clinical supervisor. The issue of managing underperformance alongside personal development further highlights this tension and indicates again that having two different 
supervisors might offer a solution; like experienced by junior doctors in the UK, who have an educational supervisor (who overseas educational development) and a clinical supervisor (who overseas clinical practice). A split role, when feasible, might be the preferred solution and when this is not an option then supervisors need training to support them to manage these challenges. The model of practice which is best is a source of continued debate. However, what is clear is that there is no 'one size fits all' for clinical supervision and all stakeholders need to consider how to make their supervision as effective as it can be and involve discussion to agree on the shared purpose of clinical supervision.

\section{Limitations}

This paper was based on evidence identified in the international literature using a rapid review, which involves a systematic search and rigorous analysis. Although in many places there was a vast amount of information, which provides strength to the findings, a rapid review necessarily pays less attention to study design and sample sizes. An additional limitation of this rapid review was that the study focused on publications in English, studies set in Western only settings and publications within the past 10 years only. Much of the data were heterogeneous in nature, and this also hindered our ability to relate the findings to specific professions and settings. However, the findings drawn from the overall themes were evident across much of the literature.

\section{CONCLUSIONS}

This review has identified the following enablers of and barriers to effective clinical supervision with regard to the organisation, the supervisor and supervisee.

Enablers included having a set place and a regular time slot for supervision to ensure it takes place. It is more likely to occur when a private space is made available and when protected time is available. Also, there needs to be some flexibility to enable staff working irregular hours such as night shift to access clinical supervision. Barriers to supervision happening were apparent when these issues of place and time were not in place.

Another key enabler identified was when the supervisory relationship was based on a positive relationship and on mutual trust. Ideally, supervisees should be offered a choice of supervisor and there should be some cultural understanding between them. When this is not the case, when the relationship lacks trust, this becomes a barrier, as does having to accept a supervisor not well matched to the supervisee and when cultural understanding is missing.

For clinical supervision to be effective, there needs to be a shared understanding of its purpose. Ideally, this is based on the individual needs of the supervisee and the focus is on enhancing knowledge and skills to support professional development and improve the service. Barriers occur when there is no agreed purpose and no agreement or conflicting views on the focus of clinical supervision.

This review identified that a range of types of supervision can be effective: one-to-one, group, internal, external and distance supervision can all offer a range of benefits. Going forward, having different types of supervision, with different people who offer different perspectives, should be considered and may overcome some of the barriers in place when only one, poorly matched, supervisor is available. Clearly, having different supervisors also overcomes the problem of having a line manager who is both the clinical supervisor and service manager and who may need to manage the needs of the service with the potentially conflicting needs to the individual. Lastly, providing training to supervisors is helpful to ensure they are supported and developed in this role and indeed also benefit from feedback themselves, without such training barriers to effective supervision may occur.

Contributors CR oversaw jointly with AK the project management and liaison with the funder, and had substantial contribution to the conception and design of the project, contribution to the reviewing, interpreting, analysing and writing of the article and final approval for important intellectual content and approval of the final version to be published. AK oversaw jointly with CR the project management and liaison with the funder, and had substantial contribution to the conception and design of the project, contribution to the reviewing, interpreting, analysing and writing of the article and final approval for important intellectual content and approval of the final version to be published. SFF had substantial contribution to the reviewing, analysing, interpreting the data and drafting of and approval for important intellectual content and approval of the final version to be published. $\mathrm{JI}$ had substantial contribution to the conception and design of the project, contribution to the reviewing, interpreting, analysing and writing of the article and final approval for important intellectual content and approval of the final version to be published.

Funding Health and Care Professions Council (HCPC) commissioned and funded a rapid review to look at the characteristics of clinical supervision in the clinical workplace (grant number NA). This paper draws down from findings of the rapid review, but was not commissioned and funded by HCPC.

Competing interests None declared.

Patient consent for publication Not applicable.

Provenance and peer review Not commissioned; externally peer reviewed.

Data availability statement Data sharing not applicable as no datasets generated and/or analysed for this study.

Supplemental material This content has been supplied by the author(s). It has not been vetted by BMJ Publishing Group Limited (BMJ) and may not have been peer-reviewed. Any opinions or recommendations discussed are solely those of the author(s) and are not endorsed by BMJ. BMJ disclaims all liability and responsibility arising from any reliance placed on the content. Where the content includes any translated material, BMJ does not warrant the accuracy and reliability of the translations (including but not limited to local regulations, clinical guidelines, terminology, drug names and drug dosages), and is not responsible for any error and/or omissions arising from translation and adaptation or otherwise.

Open access This is an open access article distributed in accordance with the Creative Commons Attribution Non Commercial (CC BY-NC 4.0) license, which permits others to distribute, remix, adapt, build upon this work non-commercially, and license their derivative works on different terms, provided the original work is properly cited, appropriate credit is given, any changes made indicated, and the use is non-commercial. See: http://creativecommons.org/licenses/by-nc/4.0/.

\section{ORCID iDs}

Charlotte Rothwell http://orcid.org/0000-0002-2240-3009

Amelia Kehoe http://orcid.org/0000-0002-3549-3234

Jan Illing http://orcid.org/0000-0001-6218-9775 


\section{REFERENCES}

1 Beddoe L. External supervision in social work: power, space, risk, and the search for safety. Australian Social Work 2012;65:197-213.

2 Nancarrow SA, Wade R, Moran A. Connecting practice: a practitioner centred model of supervision. Clinical Governance: An International Journal 2014;19:235-52.

3 Proctor B. Group supervision: a guide to creative practice. Thousand Oaks: Sage, 2008.

4 Binnie J. Structured reflection on the clinical supervision of supervisees with and without a core mental health professional background. Issues Ment Health Nurs 2011;32:584-8.

5 Kilminster SM, Jolly BC. Effective supervision in clinical practice settings: a literature review. Med Educ 2000;34:827-40.

6 Bernard JM, Goodyear RK, Bernard JM. Fundamentals of clinical supervision. 3rd edition. Massachusetts, USA: Allyn \& Bacon, 1992. ISBN: 9780205388738

7 Haby MM, Chapman E, Clark R, et al. What are the best methodologies for rapid reviews of the research evidence for evidence-informed decision making in health policy and practice: a rapid review. Health Res Policy Syst 2016;14:83.

8 Thomas J, Harden A. Methods for the thematic synthesis of qualitative research in systematic reviews. BMC Med Res Methodo 2008;8:45

9 Beddoe L. Surveillance or reflection: professional supervision in 'the Risk Society'. Br J Soc Work 2010;40:1279-96.

10 Beddoe L, Howard F. Interprofessional supervision in social work and psychology: mandates and (inter) professional relationships. Clin Superv 2012;31:178-202.

11 Love B, Sidebotham M, Fenwick J, et al. "Unscrambling what's in your head": a mixed method evaluation of clinical supervision for midwives. Women and Birth 2017;30:271-81.

12 Dawber C. Reflective practice groups for nurses: a consultation liaison psychiatry nursing initiative: part 2--the evaluation. Int $J$ Ment Health Nurs 2013;22:241-8.

13 O'Donoghue K, Tsui M-s, Ming-sum T. Social work supervision research (1970-2010): the way we were and the way ahead. Br J Soc Work 2015;45:616-33.

14 Morgan S. Supervising the highly performing general practice registrar. Clin Teach 2014;11:53-7.

15 Brink P, Bäck-Pettersson S, Sernert N. Group supervision as a means of developing professional competence within pre-hospital care. Int Emerg Nurs 2012;20:76-82.

16 Davis C, Burke L. The effectiveness of clinical supervision for a group of ward managers based in a district general Hospital: an evaluative study. J Nurs Manag 2012;20:782-93.

17 Cross W, Moore A, Ockerby S. Clinical supervision of general nurses in a busy medical ward of a teaching hospital. Contemp Nurse 2010;35:245-53.

18 Greenway JC, Entwistle VA, Termeulen R. Health visitor professional education and post-qualification clinical supervision: how well does it equip practitioners for dealing with ethical tensions associated with promoting the public health agenda to individual clients? Prim Health Care Res Dev 2013;14:90-102.

19 Rouzaud-Laborde C, Damery L, Cestac P, et al. Mentoring and supervising clinical pharmacist students at patients' bedside: which benefits? J Eval Clin Pract 2016;22:4-9.

20 Paulin V. Professional supervision in dietetics: A focus group study investigating New Zealand dietitians' understanding and experience of professional supervision and their perception of its value in dietetic practice. Nutrition Dietetics 2010;67:106-11.

21 Samuel S, Thompson H. Critical reflection: a general practice support group experience. Aust J Prim Health 2018;24:204-7.

22 Koivu A, Saarinen PI, Hyrkas K. Stress relief or practice development: varied reasons for attending clinical supervision. $J$ Nurs Manag 2011;19:644-54.

23 Koivu A, Saarinen PI, Hyrkas K. Who benefits from clinical supervision and how? the association between clinical supervision and the work-related well-being of female Hospital nurses. J Clin Nurs 2012;21:2567-78.

24 Bondas T. Nursing leadership from the perspective of clinical group supervision: a paradoxical practice. J Nurs Manag 2010;18:477-86.

25 Snowdon DA, Millard G, Taylor NF. Effectiveness of clinical supervision of physiotherapists: a survey. Aust Health Rev 2015;39:190-6.

26 Snowdon DA, Leggat SG, Taylor NF. Does clinical supervision of healthcare professionals improve effectiveness of care and patient experience? A systematic review. BMC Health Serv Res 2017;17:786.

27 McMahon A, Errity D. From new vistas to life lines: psychologists' satisfaction with supervision and confidence in supervising. Clin Psychol Psychother 2014;21:264-75.
28 Sexton A, Hodgkin L, Bogwitz M, et al. A model for peer experiential and reciprocal supervision (peers) for genetic Counselors: development and preliminary evaluation within clinical practice. $J$ Genet Couns 2013:22:175-87.

29 Shea SE, Goldberg S, Weatherston DJ. A community mental health professional development model for the expansion of reflective practice and supervision: evaluation of a pilot training series for infant mental health professionals. Infant Ment Health J 2016;37:653-69.

30 Redpath AA, Gill SD, Finlay N, et al. Public sector physiotherapists believe that staff supervision should be broad ranging, individualised, structured, and based on needs and goals: a qualitative study. J Physiother 2015;61:210-6.

31 Hair HJ. Supervision conversations about social justice and social work practice. Journal of Social Work 2015;15:349-70.

32 Wilson HMN, Davies JS, Weatherhead S. Trainee therapists experiences of supervision during training: a Meta-synthesis. Clin Psychol Psychother 2016;23:340-51.

33 Spackman R, Toogood H, Kerridge J, et al. Trainee experiences of intellectual disability psychiatry and an innovative leaderless support group: a qualitative study. BJPsych Bull 2017;41:228-33.

34 Kutzsche S, Kutzsche H, Dornan T. What do paediatric trainees require from their clinical supervisors? Acta Paediatr 2014;103:e169-72.

35 Dawson M, Phillips B, Leggat SG. Effective clinical supervision for regional allied health professionals - the supervisee's perspective. Aust Health Rev 2012;36:92-7.

36 Wallbank S. Effectiveness of individual clinical supervision for midwives and doctors in stress reduction: findings from a pilot study. Evidence Based Midwifery 2010;8:65-70.

37 Phillips S, Berman S, Hector S, et al. An evaluation study of a clinical supervision programme. Dental Nursing 2012;8:794-9.

38 Milne D. Can we enhance the training of clinical supervisors? a national pilot study of an evidence-based approach. Clin Psychol Psychother 2010;17:321-8.

39 Martin P, Kumar S, Lizarondo L, et al. Enablers of and barriers to high quality clinical supervision among occupational therapists across Queensland in Australia: findings from a qualitative study. BMC Health Serv Res 2015;15:1-8.

40 Cox DL, Araoz G. The experience of therapy supervision within a UK multi-centre randomized controlled trial. Learning in Health Social Care 2009;8:301-14.

41 Gagliardi AR, Wright FC. Exploratory evaluation of surgical skills mentorship program design and outcomes. J Contin Educ Health Prof 2010;30:51-6.

42 Martin P, Copley J, Tyack Z. Twelve tips for effective clinical supervision based on a narrative literature review and expert opinion. Med Teach 2014;36:201-7.

43 Brown J, Nestel D, Clement T, et al. The supervisory encounter and the senior GP trainee: managing for, through and with. Med Educ 2018;52:192-205.

44 Palmer-Olsen L, Gold LL, Woolley SR. Supervising emotionally focused therapists: a systematic research-based model. J Marital Fam Ther 2011;37:411-26.

45 Ellis MV, Berger L, Hanus AE. Inadequate and harmful clinical supervision: testing a revised framework and assessing occurrence. Counseling Psychologist 2014;42:434-72.

46 Annan J, Ryba K. Networks of professional supervision. Sch Psychol Q 2013;28:170-82.

47 West A. Supervising counsellors and psychotherapists who work with trauma: a Delphi study. Br J Guid Counc 2010;38:409-30.

48 Lalani N, Griffith KA, Jones RD, et al. Mentorship experiences of early-career academic radiation oncologists in North America. Int $J$ Radiat Oncol Biol Phys 2018;101:732-40.

49 McLaren P, Patel A, Trafford P, et al. GP trainers' experience of managing a trainee in difficulty: a qualitative study. Educ Prim Care 2013;24:363-71.

50 Daveson B, Kennelly J. Reflections regarding Australian music therapy supervision: guidance and recommendations for establishing internal and external supervisory arrangements aided by cross-national reflection. Australian Journal of Music Therapy 2011;22:24-34.

51 Jelinek GA, Weiland TJ, Mackinlay C. Supervision and feedback for junior medical staff in Australian emergency departments: findings from the emergency medicine capacity assessment study. BMC Med Educ 2010;10:74.

52 Rodwell J, McWilliams J, Gulyas A. The impact of characteristics of nurses' relationships with their supervisor, engagement and trust, on performance behaviours and intent to quit. J Adv Nurs 2017;73:190-200. 
53 Ostergren JA, Aguilar SM. A survey of speech-language pathology assistants (SLPAs) in California: current trends in demographics, employment, supervision, and training. CICSD 2012;39:121-36.

54 Gray TG, Hood G, Farrell T. The results of a survey highlighting issues with feedback on medical training in the United Kingdom and how a smartphone APP could provide a solution. BMC Res Notes 2015;8:653.

55 Bomba J. Psychotherapy supervision as viewed from psychodynamic standpoint. Psychoterapia 2011;4:45-9.

56 Gonge H, Buus N. Individual and workplace factors that influence psychiatric nursing staff's participation in clinical supervision: a survey study and prospective longitudinal registration. Issues Ment Health Nurs 2010;31:345-54

57 Dilworth S, Higgins I, Parker V, et al. Finding a way forward: a literature review on the current debates around clinical supervision. Contemp Nurse 2013;45:22-32.

58 Saxby C, Wilson J, Newcombe P. Can clinical supervision sustain our workforce in the current healthcare landscape? findings from a Queensland study of allied health professionals. Aust Health Rev 2015;39:476-82.

59 Balmer D, D'Alessandro D, Risko W, et al. How mentoring relationships evolve: a longitudinal study of academic pediatricians in a physician educator faculty development program. $J$ Contin Educ Health Prof 2011;31:81-6.

60 Kenny A, Allenby A. Implementing clinical supervision for Australian rural nurses. Nurse Educ Pract 2013;13:165-9.

61 Chiller P, Crisp BR. Professional supervision: a workforce retention strategy for social work? Australian Social Work 2012;65:232-42.

62 Allan H. Mentoring overseas nurses: barriers to effective and nondiscriminatory mentoring practices. Nurs Ethics 2010;17:603-13.

63 Gibson JA, Grey IM, Hastings RP. Supervisor support as a predictor of burnout and therapeutic self-efficacy in therapists working in ABA schools. J Autism Dev Disord 2009;39:1024-30.

64 O'Connell B, Ockerby CM, Johnson S, et al. Team clinical supervision in acute hospital wards: a feasibility study. West $J$ Nurs Res 2013;35:330-47.

65 Rodwell J, Martin A. The importance of the supervisor for the mental health and work attitudes of Australian aged care nurses. Int Psychogeriatr 2013;25:382-9.

66 Brunetto Y, Farr-Wharton R, Shacklock K. Supervisor-subordinate communication relationships, role ambiguity, autonomy and affective commitment for nurses. Contemp Nurse 2011;39:227-39.

67 Brunetto Y, Farr-Wharton R, Shacklock K. The impact of supervisorsubordinate relationships on nurses' ability to solve workplace problems: implications for their commitment to the organization. Adv Health Care Manag 2011;10:215-37.

68 Pohl S, Galletta M. The role of supervisor emotional support on individual job satisfaction: a multilevel analysis. Appl Nurs Res 2017;33:61-6.

69 Bethell J, Chu CH, Wodchis WP, et al. Supportive supervision and staff intent to turn over in long-term care homes. Gerontologist 2018:58:953-9.

70 Brunetto Y, Shriberg A, Farr-Wharton R, et al. The importance of supervisor-nurse relationships, teamwork, wellbeing, affective commitment and retention of North American nurses. J Nurs Manag 2013;21:827-37.

71 Dunsmuir S, Lang J, Leadbetter J. Current trends in educational psychology supervision in the UK. Educational and Child Psychology 2015;32:8-21.

72 Pack M. Two sides to every story: a phenomenological exploration of the meanings of clinical supervision from supervisee and supervisor perspectives. J Soc Work Pract 2012;26:163-79.

73 O'Connor T. Developing 'home-grown' supervisors. Kai Tiaki Nursing New Zealand 2012;18:30-1.

74 Noelker LS, Ejaz FK, Menne HL, et al. Factors affecting frontline workers' satisfaction with supervision. J Aging Health 2009;21:85-101.

75 Gonge $\mathrm{H}$, Buus N. Is it possible to strengthen psychiatric nursing staff's clinical supervision? RCT of a meta-supervision intervention. J Adv Nurs 2015;71:909-21.

76 da Silva Pinheiro GM, de Carvalho Macedo APM, Nunes da Costa NMV. Collaborative supervision and professional development in nursing. Revista de Enfermagem Referência 2014;4:101-9.

77 Pack M. 'Unsticking the stuckness': a qualitative study of the clinical supervisory needs of early-career health social workers: table 1. Br J Soc Work 2015;45:1821-36.

78 Beddoe L, Davys AM, Adamson C. 'Never trust anybody who says "i don't need supervision"': practitioners' beliefs about social worker resilience. Practice 2014;26:113-30.

79 Rodwell J, Brunetto Y, Demir D, et al. Abusive supervision and links to nurse intentions to quit. J Nurs Scholarsh 2014;46:357-65.
80 Carless SA, Robertson K, Willy J, et al. Successful postgraduate placement experiences: what is the influence of job and supervisor characteristics? Aust Psychol 2012;47:156-64.

81 Claridge JA, Carter JW, McCoy AM, et al. In-House direct supervision by an attending is associated with differences in the care of patients with a blunt splenic injury. Surgery 2011;150:718-26.

82 Farnan JM, Johnson JK, Meltzer DO, et al. Strategies for effective on-call supervision for internal medicine residents: the superb/ safety model. J Grad Med Educ 2010;2:27.

83 Bryant AL, Aizer Brody A, Perez A, et al. Development and implementation of a peer mentoring program for early career gerontological faculty. J Nurs Scholarsh 2015;47:258-66.

84 Bulman C, Forde-Johnson C, Griffiths A, et al. The development of peer reflective supervision amongst nurse educator colleagues: an action research project. Nurse Educ Today 2016;45:148-55.

85 Bucky SF, Marques S, Daly J, et al. Supervision characteristics related to the supervisory working alliance as rated by doctorallevel supervisees. Clin Superv 2010;29:149-63.

86 Davies JM, Edgar S, Debenham J. A qualitative exploration of the factors influencing the job satisfaction and career development of physiotherapists in private practice. Man Ther 2016;25:56-61.

87 Danielsson A, Sundin-Andersson C, Hov R, et al. Norwegian and Swedish preceptors' views of their role before and after taking part in a group supervision program. Nurs Health Sci 2009;11:107-13.

88 Artinian H, Mehra S, Yang S. Peer mentorship during pediatric residency: a pilot study. Journal of Investigative Medicine 2014;62:248.

89 Brody AA, Edelman L, Siegel EO, et al. Evaluation of a peer mentoring program for early career gerontological nursing faculty and its potential for application to other fields in nursing and health sciences. Nurs Outlook 2016:64:332-8.

90 Griggs C. Mentoring community-based trainee assistant practitioners: a case study. Br J Community Nurs 2012;17:328-32.

91 Perron NJ, Sommer J, Hudelson P, et al. Clinical supervisors perceived needs for teaching communication skills in clinical practice. Med Teach 2009;31:e316-22.

92 Szabo S, Lloyd B, McKellar D, et al. 'Having a mentor helped me with difficult times': a trainee-run mentoring project. Australas Psychiatry 2019;27:230-3.

93 Webb J, Brightwell A, Sarkar P, et al. Peer mentoring for core medical trainees: uptake and impact. Postgrad Med $\mathrm{J}$ 2015;91:188-92.

94 Simpson-Southward C, Waller G, Hardy GE. How do we know what makes for "best practice" in clinical supervision for psychological therapists? A content analysis of supervisory models and approaches. Clin Psychol Psychother 2017;24:1228-45.

95 Gonge H, Buus N. Exploring organizational barriers to strengthening clinical supervision of psychiatric nursing staff: a longitudinal controlled intervention study. Issues Ment Health Nurs 2016;37:332-43.

96 McBride AB, Campbell J, Woods NF, et al. Building a mentoring network. Nurs Outlook 2017;65:305-14.

97 O'Connell JE, McKay EA, Profile MEA. Profile, practice and perspectives of occupational therapists in community mental health teams in Ireland. British Journal of Occupational Therapy 2010;73:219-28.

98 Henderson A, Tyler S. Facilitating learning in clinical practice: evaluation of a trial of a supervisor of clinical education role. Nurse Educ Pract 2011;11:288-92.

99 Laschober TC, de Tormes Eby LT, Sauer JB. Clinical supervisor and counselor perceptions of clinical supervision in addiction treatment. $J$ Addict Dis 2012;31:382-8.

100 Goldszmidt M, Faden L, Dornan T, et al. Attending physician variability: a model of four supervisory styles. Acad Med 2015;90:1541-6.

101 Buus N, Gonge H. Empirical studies of clinical supervision in psychiatric nursing: a systematic literature review and methodological critique. Int J Ment Health Nurs 2009;18:250-64.

102 Akhigbe T, Zolnourian A, Bulters D. Mentoring models in neurosurgical training: review of literature. Journal of Clinical Neuroscience 2017;45:40-3.

103 Koivu A, Hyrkas K, Saarinen PI. Who attends clinical supervision? the uptake of clinical supervision by hospital nurses. J Nurs Manag 2011;19:69-79.

104 Harrison N, Lyons C, Baguley C, et al. An educational evaluation of supervisor and mentor experiences when supporting primary care graduate mental health workers. J Psychiatr Ment Health Nurs 2009:16:416-23. 
105 Iwanicki S, Peterson C. An exploratory study examining current assessment Supervisory practices in professional psychology. $J$ Pers Assess 2017;99:165-74.

106 Elfering A, Gerhardt C, Grebner S, et al. Exploring SupervisorRelated job resources as mediators between supervisor conflict and job attitudes in hospital employees. Saf Health Work 2017;8:19-28.

107 Cheung G, Stephan A. Supervision: 'a random bag of arrangements'? Perspectives from psychiatrists on how to improve clinical teaching. Australas Psychiatry 2017;25:510-3.

108 Lewis KL, Erby LAH, Bergner AL, et al. The dynamics of a genetic counseling peer supervision group. J Genet Couns 2017;26:532-40.

109 Henderson A, Ossenberg C, Tyler S. 'What matters to graduates ${ }^{\mathrm{TM}}$ : an evaluation of a structured clinical support program for newly graduated nurses. Nurse Educ Pract 2015;15:225-31.

110 Churchill J, Rashid R. Supervision of urological surgical trainees: what are the issues and how can we improve? BJU International 2017; 119:52.

111 Kilbertus S, Pardhan K, Bandiera G, et al. P079: transition to practice: evaluating the need for formal training in supervision and assessment techniques among senior emergency medicine residents and new to practice emergency physicians. CJEM 2018;20:S84-5

112 Clark T, White SJ. Becoming an effective pharmacy supervisor. American Journal of Health-System Pharmacy 2010;67:977-80.

113 McAuliffe E, Daly M, Kamwendo F, et al. The critical role of supervision in retaining staff in obstetric services: a three country study. PLoS One 2013;8:e58415.

114 Davys AM, Beddoe L. Interprofessional learning for supervision: 'taking the blinkers off'. Learning in Health \& Social Care 2009;8:58-69.

115 Jarrett P, Barlow J. Clinical supervision in the provision of intensive home visiting by health visitors. Community Practitioner 2014;87:32-6.

116 Colthart I, Bagnall G, Evans A, et al. The effectiveness of selfassessment on the identification of learner needs, learner activity, and impact on clinical practice: BEME guide No. 10. Med Teach 2008;30:124-45.
117 Dawber C. Reflective practice groups for nurses: a consultation liaison psychiatry nursing initiative: part 1 - the model reflective practice groups for nurses: a consultation liaison psychiatry nursing initiative: Part 1 - the model. International Journal of Mental Health Nursing 2013;22:135-44.

118 Grant J, Schofield MJ, Crawford S. Managing difficulties in supervision: Supervisors' perspectives. J Couns Psychol 2012;59:528-41.

119 Fellows S, Brightwell A, Eisen S, et al. G264 Mentoring for Paediatricians - need and support for a National Framework. Arch Dis Child 2014;99:A114.

120 Taylor C. Receiving group clinical supervision: a phenomenological study. Br J Nurs 2013;22:861-6.

121 Milne D, Reiser RP. A rationale for evidence-based clinical supervision. J Contemp Psychother 2012;42:139-49.

122 O'Connor T. Developing'home-grown'supervisors. Kai Tiaki: Nursing New Zealand 2012;18:30.

123 Love B. Beyond the horizon-clinical supervision-a journey through reflection. Women and Birth 2011;24:S40.

124 Cross WM, Moore AG, Sampson T, et al. Implementing clinical supervision for ICU outreach nurses: a case study of their journey. Australian Critical Care 2012;25:263-70.

125 McMahon A. Four guiding principles for the supervisory relationship. Reflective Practice 2014;15:333-46.

126 Baines D, Charlesworth S, Turner D, et al. Lean social care and worker identity: the role of outcomes, supervision and mission. Critical Social Policy 2014;34:433-53.

127 O'Donoghue K. Windows on the Supervisee experience: an exploration of Supervisees' supervision histories. Australian Social Work 2012;65:214-31.

128 Buus N, Angel S, Traynor M, et al. Psychiatric nursing staff members' reflections on participating in group-based clinical supervision: A semistructured interview study. Int J Ment Health Nurs 2011;20:95-101.

129 Snowdon DA, Sargent M, Williams CM, et al. Effective clinical supervision of allied health professionals: a mixed methods study. BMC Health Serv Res 2020;20:1-11. 